Jean Dolbeault · Maria J. Esteban · Eric Séré

\title{
General results on the eigenvalues of operators with gaps, arising from both ends of the gaps. Application to Dirac operators
}

\author{
Received September 20, 2005
}

\begin{abstract}
This paper is concerned with an extension and reinterpretation of previous results on the variational characterization of eigenvalues in gaps of the essential spectrum of self-adjoint operators. We state two general abstract results on the existence of eigenvalues in the gap and a continuation principle. Then these results are applied to Dirac operators in order to characterize simultaneously eigenvalues corresponding to electronic and positronic bound states.
\end{abstract}

\section{Introduction}

In [4] we proved an abstract result on the variational characterization of the eigenvalues of operators with gaps in the essential spectrum. Such a result was designed to deal with nonpositive perturbations of a fixed self-adjoint operator with a gap in its essential spectrum but without eigenvalues. In that case, the "branching" of the potential "pulls down" eigenvalues from the right hand side of the gap. In other words, these eigenvalues emerge from the right end of the gap when the coupling is turned on. Here we address the general case of a perturbation with negative and positive parts, so that eigenvalues can emerge simultaneously from the left and right hand sides of the gap. We observe that a simple extension of the general abstract result proved in [4] allows us to treat much more general cases.

For a historical overview of the subject, we refer the reader to the introduction of [4], in which an extended review of the literature on eigenvalues in gaps of the essential spectrum is presented. Some relevant physics papers dealing with this problem are [5, 10, 15, 2] (see also the references therein). On the mathematical side we can quote (in chronological order) [6, 8, 7, 3, 4].

Let $\mathcal{H}$ be a Hilbert space with scalar product $(\cdot, \cdot)$, and $A: D(A) \subset \mathcal{H} \rightarrow \mathcal{H}$ be a self-adjoint operator. We denote by $\mathcal{H}^{\prime}$ the dual of $\mathcal{H}$ and by $\mathcal{F}(A)$ the form-domain of $A$. Let $\mathcal{H}_{+}, \mathcal{H}_{-}$be two orthogonal Hilbert subspaces of $\mathcal{H}$ such that $\mathcal{H}=\mathcal{H}_{+} \oplus \mathcal{H}_{-}$. We denote by $\Lambda^{+}, \Lambda^{-}$the projectors on $\mathcal{H}_{+}, \mathcal{H}_{-}$. We assume the existence of a core $F$ (i.e. a subspace of $D(A)$ which is dense for the norm $\left.\|\cdot\|_{D(A)}\right)$ such that:

Partially supported by European Programs HPRN-CT \# 2002-00277 \& 00282.

J. Dolbeault, M. J. Esteban, E. Séré: Ceremade (UMR CNRS no. 7534), Université Paris-Dauphine, Place de Lattre de Tassigny, 75775 Paris Cedex 16, France; e-mail: \{dolbeaul, esteban, sere\}@ ceremade.dauphine.fr, web: http://www.ceremade.dauphine.fr/ $\sim$ dolbeaul, $\sim$ esteban, $\sim$ sere 
(i) $F_{+}=\Lambda^{+} F$ and $F_{-}=\Lambda^{-} F$ are two subspaces of $\mathcal{F}(A)$.

(ii $\left.{ }^{-}\right) a^{-}:=\sup _{x_{-} \in F_{-} \backslash\{0\}}\left(x_{-}, A x_{-}\right) /\left\|x_{-}\right\|_{\mathcal{H}}^{2}<+\infty$.

(ii $\left.{ }^{+}\right) a^{+}:=\inf _{x_{+} \in F_{+} \backslash\{0\}}\left(x_{+}, A x_{+}\right) /\left\|x_{+}\right\|_{\mathcal{H}}^{2}>-\infty$.

We consider the sequences of min-max and max-min levels $\left(\lambda_{k}^{+}\right)_{k \geq 1}$ and $\left(\lambda_{k}^{-}\right)_{k \geq 1}$ defined by

$$
\begin{aligned}
& \lambda_{k}^{+}:=\inf _{\begin{array}{c}
V \text { subspace of } \\
\operatorname{dim} V=k
\end{array}} \sup _{+} \sup _{x \in\left(V \oplus F_{-}\right) \backslash\{0\}} \frac{(x, A x)}{\|x\|_{\mathcal{H}}^{2}}, \\
& \lambda_{k}^{-}:=\sup _{\begin{array}{c}
V \text { subspace of } F_{-} \\
\operatorname{dim} V=k
\end{array}} \inf _{x \in\left(V \oplus F_{+}\right) \backslash\{0\}} \frac{(x, A x)}{\|x\|_{\mathcal{H}}^{2}} .
\end{aligned}
$$

The sequences $\left(\lambda_{k}^{+}\right)_{k \geq 1}$ and $\left(\lambda_{k}^{-}\right)_{k \geq 1}$ are respectively nondecreasing and nonincreasing. As a consequence of their definitions we have :

$$
\lambda_{k}^{+} \geq \max \left\{a^{-}, a^{+}\right\} \quad \text { and } \quad \lambda_{k}^{-} \leq \min \left\{a^{-}, a^{+}\right\} \quad \text { for all } k \geq 1 .
$$

Let $b^{-}:=\inf \left\{\sigma_{\text {ess }}(A) \cap\left(a^{-}, \infty\right)\right\}$ and $b^{+}:=\sup \left\{\sigma_{\text {ess }}(A) \cap\left(-\infty, a^{+}\right)\right\}$, and consider the two cases

(iii-) $k_{0}^{+}:=\min \left\{k \geq 1: \lambda_{k}^{+}>a^{-}\right\}$,

$\left(\right.$ iii $\left.^{+}\right) k_{0}^{-}:=\min \left\{k \geq 1: \lambda_{k}^{-}<a^{+}\right\}$.

Theorem 1. If (i)-(ii $\left.{ }^{-}\right)-\left(\mathrm{iii}^{-}\right)$hold, then for any $k \geq k_{0}^{+}$, either $\lambda_{k}^{+}$is the $\left(k-k_{0}^{+}+1\right)$-th eigenvalue of $A$ in the interval $\left(a^{-}, b^{-}\right)$or it is equal to $b^{-}$. If (i)-(ii $\left.{ }^{+}\right)$-(iii $\left.{ }^{+}\right)$hold, then for any $k \geq k_{0}^{-}$, either $\lambda_{k}^{-}$is the $\left(k-k_{0}^{-}+1\right)$-th eigenvalue of $A$ (in reverse order) in the interval $\left(b^{+}, a^{+}\right)$or it is equal to $b^{+}$.

Eigenvalues are counted with multiplicity, and the order has no meaning if, for instance, $\lambda_{k}^{+}=\lambda_{k+1}^{+}$. The above result does not state anything about the possible eigenvalues of $A$ in the interval $\left[a^{+}, a^{-}\right]$if $a^{-} \geq a^{+}$. We will extensively comment on this in Section 2 and explain how the abstract result of [4] implies Theorem 1 and a continuation result. In Section 3 we will address the particular case in which the operator $A$ is of the form $H_{0}+V$, where $H_{0}$ is the free Dirac operator and $V$ is an electrostatic scalar potential.

\section{Abstract results}

Theorem 1.1 in [4] can be stated as follows:

Under the assumptions of Theorem 1 if $\lambda_{1}^{+}>a^{-}$, then all eigenvalues in $\left(a^{-}, b^{-}\right)$ are given by the min-max levels $\lambda_{k}^{+}$as long as they take their values in $\left(a^{-}, b^{-}\right)$(and otherwise, $\lambda_{k}^{+}=b^{-}$).

This result dealt with the family of eigenvalues $\left\{\lambda_{k}^{+}\right\}_{k}$ and only in the case $k_{0}^{+}=1$. Nothing was said on eigenvalues below $a^{+}$. The result in [4] already covered all cases 
corresponding to a Dirac operator with a potential given by a positive Coulomb singularity. Here, by considering the case $k_{0}^{+} \geq 1$ and by considering the levels $\lambda_{k}^{-}$as well, we extend the method to a framework with interesting physical applications.

The proof for $k_{0}^{+}>1$ is similar to the proof given in [4] and we will not reproduce it here. A posteriori, passing from $k_{0}^{+}=1$ to $k_{0}^{+}>1$ is not very difficult. Consider indeed a $\left(k_{0}^{+}-1\right)$-dimensional space $V_{k_{0}^{+}-1}$ of $F^{+}$such that

$$
a^{-}=\lambda_{k_{0}^{+}-1} \leq \sup _{x \in\left(V_{k_{0}^{+}-1} \oplus F_{-}\right) \backslash\{0\}} \frac{(x, A x)}{\|x\|_{\mathcal{H}}^{2}}<\lambda_{k_{0}^{+}},
$$

and define a new decomposition $\mathcal{H}=\tilde{\mathcal{H}}^{+} \oplus \tilde{\mathcal{H}}^{-}$by setting $\tilde{\mathcal{H}}^{-}=\mathcal{H}^{-} \oplus V_{k_{0}^{+}-1}$. Then the first case of Theorem 1 reduces to Theorem 1.1 in [4].

As for the second case, note that the statement concerning the family $\left\{\lambda_{k}^{-}\right\}_{k}$ follows from that concerning $\left\{\lambda_{k}^{+}\right\}_{k}$ applied to the operator $-A$. This completes the sketch of the general ideas for the proof of Theorem 1 .

Next, as in [4], we can also consider 1-parameter families of self-adjoint operators of the form $A_{\tau}:=A_{0}+\tau V, \tau \in[0, \bar{\tau}]=\mathcal{I}, V$ being a bounded scalar potential. In this case, it would be interesting to prove (iii ${ }^{ \pm}$) for all $A_{\tau}$ knowing that $A_{0}$ satisfies it and having some spectral information on $A_{\tau}$.

More precisely, let $A_{0}: D\left(A_{0}\right) \subset \mathcal{H} \rightarrow \mathcal{H}$ be a self-adjoint operator. Let $\mathcal{H}_{+}, \mathcal{H}_{-}$, $\Lambda^{+}$and $\Lambda^{-}$be defined as in Section 1. Assume further that there is a space $F \subset \mathcal{H}$ such that, for all $\tau \in \mathcal{I}, F$ is a core for $A_{\tau}$ and the following hypotheses hold:

(j) $F_{+}=\Lambda^{+} F$ and $F_{-}=\Lambda^{-} F$ are two subspaces of $\mathcal{F}\left(A_{\tau}\right)$.

$\left(\mathrm{jj}^{-}\right)$There is $a^{-} \in \mathbb{R}$ such that $\sup _{\tau \in \mathcal{I}, x_{-} \in F_{-} \backslash\{0\}}\left(x_{-}, A_{\tau} x_{-}\right) /\left\|x_{-}\right\|_{\mathcal{H}}^{2} \leq a^{-}$.

$\left(\mathrm{jj}^{+}\right)$There is $a^{+} \in \mathbb{R}$ such that $\inf _{\tau \in \mathcal{I}, x_{+} \in F_{+} \backslash\{0\}}\left(x_{+}, A_{\tau} x_{+}\right) /\left\|x_{+}\right\|_{\mathcal{H}}^{2} \geq a^{+}$.

Define the numbers $\left(\lambda_{k}^{\tau,+}\right)_{k \geq 1}$ and $\left(\lambda_{k}^{\tau,-}\right)_{k \geq 1}$ as in 11-2 by

$$
\begin{aligned}
& \lambda_{k}^{\tau,+}=\inf _{\begin{array}{c}
V \text { subspace of } \\
\operatorname{dim} V=k
\end{array}} \sup _{+} \sup _{x \in\left(V \oplus F_{-}\right) \backslash\{0\}} \frac{\left(x, A_{\tau}\right)}{\|x\|_{\mathcal{H}}^{2}}, \\
& \lambda_{k}^{\tau,-}=\sup _{\begin{array}{c}
V \text { subspace of } F_{-} \\
\operatorname{dim} V=k
\end{array}} \inf _{x \in\left(V \oplus F_{+}\right) \backslash\{0\}} \frac{\left(x, A_{\tau}\right)}{\|x\|_{\mathcal{H}}^{2}} .
\end{aligned}
$$

With the definitions

$$
\begin{array}{ll}
a_{1}^{-}:=\inf _{\tau \in \mathcal{I}}\left[\inf \left(\sigma\left(A_{\tau}\right) \cap\left(a^{-},+\infty\right)\right)\right], & b^{-}:=\inf \left(\sigma_{\mathrm{ess}}\left(A_{0}\right) \cap\left(a^{-},+\infty\right)\right), \\
a_{1}^{+}:=\sup _{\tau \in \mathcal{I}}\left[\sup \left(\sigma\left(A_{\tau}\right) \cap\left(-\infty, a^{+}\right)\right)\right], & b^{+}:=\sup \left(\sigma_{\mathrm{ess}}\left(A_{0}\right) \cap\left(-\infty, a^{+}\right)\right),
\end{array}
$$

we obtain the following continuation principle. 
Theorem 2. Under the above assumptions

- If $\lambda_{k_{0}^{+}}^{0,+}>a^{-}$for some $k_{0}^{+} \geq 1$, and $a_{1}^{-}>a^{-}$, then for all $k \geq k_{0}^{+}$and all $\tau \in \mathcal{I}$, the numbers $\lambda_{k}^{\tau,+}$ are either eigenvalues of $A_{0}+\tau V$ in $\left(a^{-}, b^{-}\right)$, or $\lambda_{k}^{\tau,+}=b^{-}$.

- If $\lambda_{k_{0}^{-}}^{0,-}<a^{+}$for some $k_{0}^{-} \geq 1$, and $a_{1}^{+}<a^{+}$, then for all $k \geq k_{0}^{-}$and all $\tau \in \mathcal{I}$, the numbers $\lambda_{k}^{\tau,-}$ are either eigenvalues of $A_{0}+\tau V$ in $\left(b^{+}, a^{+}\right)$, or $\lambda_{k}^{\tau,-}=b^{+}$.

Exactly as in [4], one can prove this result for a class of more general (unbounded) potentials $V$ using a truncation argument and then passing to the limit in the truncation parameter. This applies to the perturbation of the free Dirac operator studied in Section 3 by potentials with Coulomb singularites. We refer the interested reader to [4] for more details.

Proof of Theorem 2. Assumptions (i), (ii $\left.{ }^{ \pm}\right)$of Theorem 1 follow from (j), (jj $\left.{ }^{ \pm}\right)$. Because of the boundedness of $V$, the maps $\mathcal{I} \ni \tau \mapsto \lambda_{k_{0}^{ \pm}}^{\tau, \pm}$ are continuous. The sets

$$
P_{k_{0}}^{+}:=\left\{\tau \in \mathcal{I}: \lambda_{k_{0}}^{\tau,+} \geq a_{1}^{-}\right\}, \quad P_{k_{0}}^{-}:=\left\{\tau \in \mathcal{I}: \lambda_{k_{0}}^{\tau,-} \leq a_{1}^{+}\right\}
$$

are thus closed in $\mathcal{I}$, and the sets

$$
Q_{k_{0}}^{+}:=\left\{\tau \in \mathcal{I}: \lambda_{k_{0}}^{\tau,+}>a^{-}\right\}, \quad Q_{k_{0}}^{-}=\left\{\tau \in \mathcal{I}: \lambda_{k_{0}}^{\tau,-}<a^{+}\right\}
$$

are open. Obviously, $P_{k_{0}}^{ \pm} \subset Q_{k_{0}}^{ \pm}$. But if $\tau \in Q_{k_{0}}^{ \pm}$then $A_{\tau}$ satisfies (iii ${ }^{ \pm}$), so it follows from Theorem 1 that

$$
\lambda_{k}^{\tau, \pm} \in \sigma\left(A_{\tau}\right) \quad \text { for all } k \geq k_{0},
$$

hence, by our assumptions, $\tau \in P_{k_{0}}^{ \pm}$. As a consequence, $P_{k_{0}}^{ \pm}=Q_{k_{0}}^{ \pm}$, and the sets $P_{k_{0}}^{ \pm}$are both open and closed in $\mathcal{I}$. But if $\lambda_{k_{0}}^{0,+}>a^{-}$(resp. $\lambda_{k_{0}}^{0,-}<a^{+}$), then $Q_{k_{0}}^{+}$(resp. $Q_{k_{0}}^{-}$) is nonempty: it contains 0 , so $Q_{k_{0}}^{+}$(resp. $Q_{k_{0}}^{-}$) coincides with $\mathcal{I}$.

Example (A Pauli type operator). For every $v>0$ consider the operator

$$
A_{\nu}=\left(\begin{array}{cc}
1-\Delta-\frac{\nu}{|x|} & 0 \\
0 & -1+\Delta+\frac{\nu}{|x|}
\end{array}\right)
$$

on $L^{2}\left(\mathbb{R}^{3}, \mathbb{C}\right)^{2}$. This operator is self-adjoint with domain $H^{2}\left(\mathbb{R}^{3}, \mathbb{C}\right)^{2}$ and form-domain $H^{1}\left(\mathbb{R}^{3}, \mathbb{C}\right)^{2}$. An easy analysis shows that for all $v>0, A_{v}$ has two families of eigenvalues:

$$
E_{v, n}^{-}=-1+\frac{v^{2}}{4 n^{2}}, \quad E_{v, n}^{+}=1-\frac{v^{2}}{4 n^{2}}, \quad n \geq 1,
$$

and moreover $a_{v}^{ \pm}=E_{v, 1}^{ \pm}$. Furthermore, for all $k \geq 1$,

$$
\lambda_{v, k}^{ \pm}=E_{v, n(k)}^{ \pm} \quad \text { if and only if } \quad v \leq \sqrt{\frac{8 n^{2}}{n^{2}+1}}, n=n(k) .
$$


Notice indeed that the eigenvalues are degenerate for any $n \geq 2$, so that we have to count the levels with multiplicity and introduce $n:=n(k)$. If $v \in\left(\sqrt{8 n^{2} /\left(n^{2}+1\right)}, \sqrt{8}\right)$, then $\lambda_{\nu, k}^{ \pm}=E_{v, 1}^{\mp}$ for any $n=n(k) \geq 1$.

Hence, if $v \leq 2$, all the eigenvalues of the operator $A_{v}$ are given by the variational procedures defining the numbers $\lambda_{k}^{ \pm}$. In the interval $v \in(2, \sqrt{8})$ some (but not all) of them still have this property. These results are illustrated in Fig. 1 below.

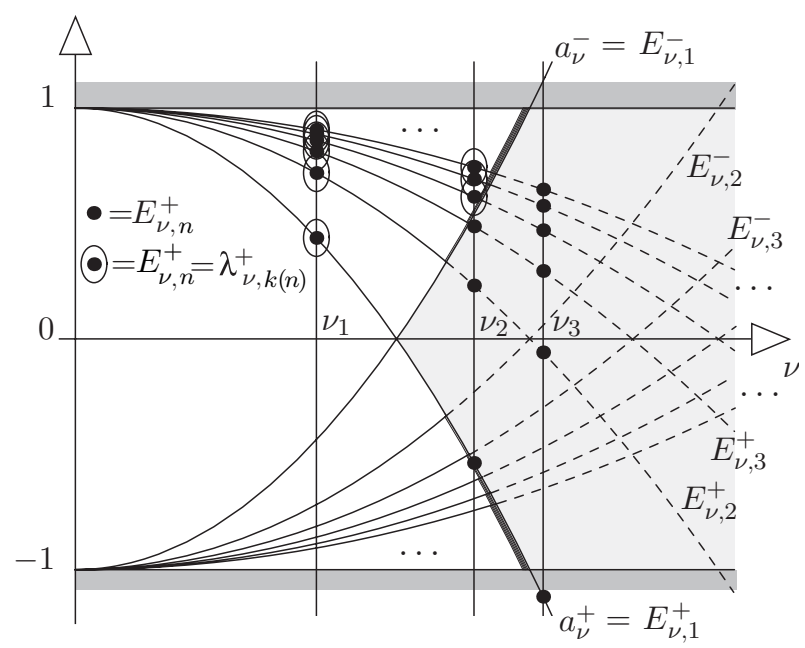

Fig. 1. Depending on the values of $v$, all eigenvalues are achieved by the two families of levels $\lambda_{\nu, k}^{+}$and $\lambda_{\nu, k}^{-}$(case $\left.v=v_{1}<2\right)$, or only some of them (case $v=v_{2} \in(2, \sqrt{8})$ ). For $v>\sqrt{8}$ (case $\left.v=v_{3}\right)$, the gap $(-1,1)$ is contained in $\left(a^{+}, a^{-}\right)$and the method does not characterize any eigenvalue in the gap. To clarify the picture, only the family of eigenvalues $E_{v, n}^{+}$has been represented, but the family $E_{\nu, n}^{-}$is easily recovered by symmetry with respect to the horizontal axis. To take the multiplicity into account, we denote by $k(n)$ the smallest $k$ for which $\lambda_{v, k}^{+}=E_{v, n}^{+}$.

\section{Application to Dirac operators}

Let us consider the free Dirac operator

$$
H_{0}:=-i \sum_{k=1}^{3} \alpha_{k} \partial_{k}+\beta,
$$

where we have written it in physical units for which the speed of light, the mass of the electron and Planck's constant are taken equal to 1 . In the Dirac equation, $\alpha_{1}, \alpha_{2}, \alpha_{3}$ and $\beta$ are $4 \times 4$ complex matrices, whose standard form (in $2 \times 2$ blocks) is

$$
\beta=\left(\begin{array}{cc}
I & 0 \\
0 & -I
\end{array}\right), \quad \alpha_{k}=\left(\begin{array}{cc}
0 & \sigma_{k} \\
\sigma_{k} & 0
\end{array}\right) \quad(k=1,2,3),
$$


and $\sigma_{k}, k=1,2,3$, are the $2 \times 2$ complex Pauli matrices: $\sigma_{1}=\left(\begin{array}{ll}0 & 1 \\ 1 & 0\end{array}\right), \sigma_{2}=\left(\begin{array}{cc}0 & -i \\ i & 0\end{array}\right)$, $\sigma_{3}=\left(\begin{array}{cc}1 & 0 \\ 0 & -1\end{array}\right)$. Let $V$ be a scalar potential satisfying

$$
\lim _{|x| \rightarrow+\infty} V(x)=0
$$

and assume that it is continuous everywhere except at two finite sets of isolated points, $\left\{x_{i}^{+}\right\},\left\{x_{j}^{-}\right\}, i=1, \ldots, I, j=1, \ldots, J$, where

$$
\begin{aligned}
& \lim _{x \rightarrow x_{i}^{+}} V(x)=+\infty, \quad \lim _{x \rightarrow x_{i}^{+}} V(x)\left|x-x_{i}^{+}\right| \leq v_{i}, \\
& \lim _{x \rightarrow x_{j}^{-}} V(x)=-\infty, \quad \lim _{x \rightarrow x_{j}^{-}} V(x)\left|x-x_{j}^{-}\right| \geq-v_{j},
\end{aligned}
$$

with $v_{i}, v_{j} \in(0,1)$ for all $i, j$. Under the above assumptions, $H_{0}+V$ has a distinguished self-adjoint extension $A$ with domain $\mathcal{D}(A)$ such that

$$
H^{1}\left(\mathbb{R}^{3}, \mathbb{C}^{4}\right) \subset \mathcal{D}(A) \subset H^{1 / 2}\left(\mathbb{R}^{3}, \mathbb{C}^{4}\right),
$$

and the essential spectrum of $A$ is the same as that of $H_{0}$ :

$$
\sigma_{\mathrm{ess}}(A)=(-\infty,-1] \cup[1,+\infty)
$$

(see [16, 14, 11, 9]). Finally, $V$ sends $\mathcal{D}(A)$ into its dual, since (4)-(5) imply that for all $\phi \in H^{1 / 2}\left(\mathbb{R}^{3}\right)$ we have $V \phi \in H^{-1 / 2}\left(\mathbb{R}^{3}\right)$.

In this section, we shall prove the validity of a variational characterization of the eigenvalues of $H_{0}+V$ corresponding to the positive/negative spectral decomposition of the free Dirac operator $H_{0}$ :

$$
\mathcal{H}=\mathcal{H}_{+}^{f} \oplus \mathcal{H}_{-}^{f}
$$

with $\mathcal{H}_{ \pm}^{f}=\Lambda_{ \pm}^{f} \mathcal{H}$, where

$$
\begin{aligned}
& \Lambda_{+}^{f}=\chi_{(0,+\infty)}\left(H_{0}\right)=\frac{1}{2}\left(\operatorname{Id}+\frac{H_{0}}{\sqrt{1-\Delta}}\right), \\
& \Lambda_{-}^{f}=\chi_{(-\infty, 0)}\left(H_{0}\right)=\frac{1}{2}\left(\operatorname{Id}-\frac{H_{0}}{\sqrt{1-\Delta}}\right) .
\end{aligned}
$$

This will be done under conditions which are optimal for the potentials satisfying (4)-(5) using Theorems 1 and 2. As already stated in [4], the theorem is optimal in the sense that it covers the optimal range in the case of Coulomb potentials. If we consider the operator $A_{\tau}:=H_{0}+\tau V, \tau>0$, with $V$ satisfying (4)-(5), our variational characterization will provide us with all eigenvalues of $A_{\tau}$ as long as $\tau$ is not too large.

Theorem 3. Take a positive integer $k_{0}$ and any $k \geq k_{0}$ and let $A$ be the self-adjoint extension of $H_{0}+V$ defined above, where $V$ is a scalar potential satisfying (4)-(5).

- If $a^{-}<\lambda_{k_{0}}^{+}<1$, then $\lambda_{k}^{+}$is either an eigenvalue of $H_{0}+V$ in the interval $\left(a^{-}, 1\right)$, or $\lambda_{k}^{+}=1$. If additionally $V \geq 0$, then $a^{-}=1$ and $\lambda_{k}^{+}=1$. 
- If $-1<\lambda_{k_{0}}^{-}<a^{+}$, then $\lambda_{k}^{-}$is either an eigenvalue of $H_{0}+V$ in the interval $\left(-1, a^{+}\right)$ or $\lambda_{k}^{-}=-1$. If additionally $V \leq 0$, then $a^{+}=-1$ and $\lambda_{k}^{-}=-1$.

The sequences $\left(\lambda_{k}^{+}\right)_{k \geq k_{0}^{ \pm}}$and $\left(\lambda_{k}^{-}\right)_{k \geq 1}$ are respectively nondecreasing and nonincreasing. The spectrum of $A$ contained in $\mathbb{R} \backslash\left[a^{+}, a^{-}\right]$is

$$
(-\infty,-1] \cup\left\{\lambda_{k}^{\epsilon}: k \geq 1, \epsilon= \pm\right\} \cup[1,+\infty),
$$

and we do not state anything about the possible eigenvalues in the interval $\left[a^{+}, a^{-}\right]$. As we showed in the previous section, there can be operators for which all or almost all the eigenvalues lie in the interval $\left[a^{+}, a^{-}\right]$and thus, they are not given by the variational procedures defining the $\lambda_{k}^{ \pm}$,s.

Theorem 3 easily follows from Theorem 11. The details of the proof are left to the reader. The continuation argument of Theorem 2 applies. Indeed, first one has to truncate the potential at some level $M$, apply Theorem 2 and then pass to the limit when $M$ goes to $+\infty$. It is worth mentioning that by the continuation principle for the Dirac operators $H_{0}+\tau V$, with $V$ satisfying 4$]-[5]$, and the definition of $\lambda_{k}^{\tau, \pm}$,

$$
\lim _{\tau \rightarrow 0^{+}} \lambda_{k}^{\tau, \pm}= \pm 1 \quad \text { for all } k \geq 1
$$

Also notice that Talman's decomposition [15, 4], i.e. the decomposition into "upper" and "lower" two-components spinors, does not apply here, while the spectral decomposition does.

Corollary 1. Under the assumptions of Theorem 3 a sufficient condition for $\lambda_{1}^{+}$to be in the interval $\left(a^{-}, 1\right)$ is

$$
c_{1}-\frac{v}{|x|} \leq V \leq c_{2}, \quad c_{1}, c_{2} \geq 0, c_{1}+c_{2}-1<\sqrt{1-v^{2}} .
$$

Proof. It is straightforward to check that $a^{-} \leq c_{2}-1$ and

$$
\lambda_{1}^{+}(V) \geq \lambda_{1}^{+}\left(-\frac{v}{|x|}\right)-c_{1}=\lambda_{1}\left(H_{0}-\frac{v}{|x|}\right)-c_{1}=\sqrt{1-v^{2}}-c_{1} .
$$

Recall that under assumptions (4)-(5), for any $k \geq 1$, for the above result to possibly imply that $\lambda_{k}^{ \pm}$is an eigenvalue we need that

$$
\pm a^{\mp}<1 \text { and } \pm\left(\lambda_{1}^{ \pm}-a^{\mp}\right)>0 .
$$

To illustrate our results, we end this paper by giving some sufficient conditions for these inequalities to hold true. Assume that $V$ satisfies (4)-(5) and can be written as

$$
V=-\sum_{i \in I} V_{i}^{-}+\sum_{j \in J} V_{j}^{+}
$$

where the $V_{i}^{-}$'s (resp. the $V_{j}^{+}$'s) are nonnegative potentials satisfying $44-\sqrt{5}$, with a unique singularity at $x_{i}^{-}$(resp. at $x_{j}^{+}$). If

$$
v_{i}, v_{j} \in[0,2 /(\pi / 2+2 / \pi)) \quad \text { for all } i \in I, j \in J
$$


it follows from [17] and [1] that there are constants $\delta_{\ell}^{ \pm} \in(0,1)$ such that, for all $i \in I$ and $j \in J$,

$$
\begin{aligned}
\delta_{i}^{-} H_{0}-V_{i}^{-} \geq 0 \quad & \text { in } \mathcal{H}_{+}, \quad \delta_{j}^{+} H_{0}+V_{j}^{+} \leq 0 \quad \text { in } \mathcal{H}_{-}, \\
a^{-}=\sup _{\substack{e \in F_{-} \\
\|e\|_{\mathcal{H}}=1}}\left(H_{0}+V\right) & \leq \sup _{\substack{e \in F_{-} \\
\|e\| \|_{\mathcal{H}}=1}}\left(H_{0}+\sum_{j \in J} V_{j}^{+}\right) \\
& \leq\left(1-\sum_{j} \delta_{j}^{+}\right) \sup _{\substack{e \in F_{-} \\
\|e\|_{\mathcal{H}}=1}} H_{0}=\sum_{j} \delta_{j}^{+}-1 .
\end{aligned}
$$

So, $a^{-}<1$ if

$$
\sum_{j \in J} \delta_{j}^{+}<2
$$

Next, let us estimate $\lambda_{1}^{+}$. For every $e_{+} \in F_{+}$,

$$
\sup _{\substack{e \in\left[e_{+}\right] \oplus F_{-} \\\|\|_{\mathcal{H}}=1}}\left(H_{0}+V\right) \geq \sup _{\substack{e \in\left[e_{+}\right] \\\|\in\|_{\mathcal{H}}=1}}\left(H_{0}-\sum_{i \in I} V_{i}^{-}\right) \geq 1-\sum_{i} \delta_{i}^{-},
$$

and hence

$$
\lambda_{1}^{+} \geq 1-\sum_{i} \delta_{i}^{-}
$$

So, finally, $\lambda_{1}^{+}>a^{-}$if

$$
\sum_{i \in I} \delta_{i}^{-}+\sum_{j \in J} \delta_{j}^{+}<2
$$

Similar computations show that $\lambda_{1}^{-}<a^{+}$if 77 holds, and $a^{+}>-1$ if

$$
\sum_{i \in I} \delta_{i}^{-}<2
$$

Conditions (6)-81) are very restrictive. If the distances between the singularity points $x_{i}^{-}$ and $x_{j}^{+}$are taken into account and made large enough, these conditions can certainly be radically weakened when these distances become large.

\section{References}

[1] Burenkov, V. I., Evans, W. D.: On the evaluation of the norm of an integral operator associated with the stability of one-electron atoms. Proc. Roy. Soc. Edinburgh Sect. A 128, 993-1005 (1998) Zbl 0917.47057 MR 1642120

[2] Datta, S. N., Deviah, G.: The minimax technique in relativistic Hartree-Fock calculations. Pramana 30, 387-405 (1988)

[3] Dolbeault, J., Esteban, M. J., Séré, E.: Variational characterization for eigenvalues of Dirac operators. Calc. Var. Partial Differential Equations 10, 321-347 (2000) Zbl 0968.49025 MR 1767717 
[4] Dolbeault, J., Esteban, M. J., Séré, E.: On the eigenvalues of operators with gaps. Application to Dirac operators. J. Funct. Anal. 174, 208-226 (2000) Zbl 0982.47006 MR 1761368

[5] Drake, G. W. F., Goldman, S. P.: Relativistic Sturmian and finite basis set methods in atomic physics. Adv. Atomic Molecular Phys. 25, 393-416 (1988)

[6] Esteban, M. J., Séré, E.: Existence and multiplicity of solutions for linear and nonlinear Dirac problems. In: Partial Differential Equations and Their Applications. CRM Proc. Lecture Notes 12, P. C. Greiner et al. (eds.), Amer. Math. Soc. (1997) Zbl 0889.35084 MR 1479240

[7] Griesemer, M., Lewis, R. T., Siedentop, H.: A minimax principle in spectral gaps: Dirac operators with Coulomb potentials. Doc. Math. 4, 275-283 (1999) (electronic) Zbl pre01317743 MR 1701887

[8] Griesemer, M., Siedentop, H.: A minimax principle for the eigenvalues in spectral gaps. J. London Math. Soc. (2) 60, 490-500 (1999) Zbl 0952.47022 MR 1724845

[9] Klaus, M., Wüst, R.: Characterization and uniqueness of distinguished self-adjoint extensions of Dirac operators. Comm. Math. Phys. 64, 171-176 (1978-79) Zbl 0408.47022 MR 0519923

[10] Kutzelnigg, W.: Relativistic one-electron Hamiltonians "for electrons only" and the variational treatment of the Dirac equation. Chem. Phys. 225, 203-222 (1997)

[11] Nenciu, G.: Self-adjointness and invariance of the essential spectrum for Dirac operators defined as quadratic forms. Comm. Math. Phys. 48, 235-247 (1976) Zbl 0349.47014 MR 0421456

[12] Reed, M., Simon, B.: Methods of Modern Mathematical Physics. Vol. 1. Academic Press, New York (1972) Zbl 0459.46001 MR 0751959

[13] Reed, M., Simon, B.: Methods of Modern Mathematical Physics. Vol. 4. Academic Press, New York (1978) Zbl 0521.47001 MR 0493421

[14] Schmincke, U. W.: Distinguished self-adjoint extensions of Dirac operators. Math. Z. 129, 335-349 (1972) Zbl 0252.35062 MR 0326448

[15] Talman, J. D.: Minimax principle for the Dirac equation. Phys. Rev. Lett. 57, 1091-1094 (1986) MR 0854208

[16] Thaller, B.: The Dirac Equation. Texts and Monographs in Physics. Springer, Berlin (1992) Zbl 0765.47023 MR 1219537

[17] Tix, C.: Strict positivity of a relativistic Hamiltonian due to Brown and Ravenhall. Bull. London Math. Soc. 30, 283-290 (1998) Zbl 0939.35134 MR 1608118 